\title{
ALDEAS INDIAS ENTRE LA ANTROPOLOGÍA Y LA HISTORIA
}

\author{
SAURABH DUBE \\ El Colegio de México
}

En los entendimientos de India, la aldea ha sido encontrada y se ha perdido varias veces ya. ${ }^{1}$ Tan importante es la aldea como unidad de entendimiento, como los materiales para su estudio que los académicos parecen deliciosamente descubrir, sólo para, de forma incierta, perderlos y después recuperarlos nuevamente; ${ }^{2}$ de hecho, estoy a punto de unirme a este coro. ${ }^{3}$

Este artículo fue recibido por la dirección de la revista el 8 de mayo de 2013 y aceptado para su publicación el 6 de noviembre de 2013.

${ }^{1}$ Este patrón puede ser rastreado de diferentes maneras: desde las formas más tempranas de gobierno colonial que destacaban el lugar de la aldea y la historia en el ejercicio de la autoridad, hasta su eclipse en la segunda mitad del siglo XIX con la aparición de un Estado imperial que se apoderó de la casta y la etnografía como modalidades de conocimiento-poder, y hasta el redescubrimiento científico-social de la aldea en India tras la independencia, y desde la multiplicidad de estudios sobre la aldea en las décadas de 1950 y 1960 pasando por su cuestionamiento en posteriores trabajos, hasta las preocupaciones de los estudios académicos actuales. Nicholas Dirks, en particular, ha discutido los cambios en las modalidades de gobierno colonial a lo largo del siglo XIx. Véase Nicholas Dirks, Castes of Mind: Colonialism and the Making of Modern India, Princeton, Princeton University Press, 2001. Para estudios contemporáneos sobre la "cuestión de la aldea”, véase Diane Mines y Nicolas Yazgi (eds.), Village Matters: Relocating Villages in the Contemporary Anthropology of India, Nueva Delhi, Oxford University Press, 2010.

${ }^{2}$ En este sentido, la declaración clásica es, desde luego, la de M. N. Srinivas, The Remembered Village, Delhi, Oxford University Press, 1976. Véase también el simposio de revisión sobre este libro que se publicó como una edición especial de Contributions to Indian Sociology, vol. 12, núm. 1, 1978.

${ }^{3}$ A pesar de que este ensayo tiene un título un tanto prosaico, se trata de un ejercicio menos exhaustivo y bastante más sugerente, lúdico y lleno de aforismos, y al tiempo se reconoce que estos atributos son un emprendimiento serio en el estudio académico. Por ello, en lugar de citas exhaustivas he brindado referencias indicativas. 


\section{Obertura}

Mi plan original para este ensayo era centrarme en dos aldeas que habían conformado sitios destacados para los esfuerzos de proselitismo de misioneros euroamericanos en la India central colonial. La primera de estas aldeas fue Bisrampur, establecida a finales de la década de 1860 por Oscar Lohr, de la Sociedad Misionera Evangélica Alemana, y la segunda fue Balodgahan, que la Misión Menonita Americana compró en 1906. Al yuxtaponer los procesos que se centran en estos sitios buscaba explorar el lugar y el papel de la aldea, como concepto y como entidad, en elaboraciones más amplias de los involucramientos evangélicos en la región de Chhattisgarh de la India central, especialmente en la sociología política de los convertidos y los misioneros que ahí se encuentran. ${ }^{4}$

Si bien se inspiraba en cierto trabajo de campo, la discusión iba a basarse principalmente en materiales de archivo consistentes en extensas notas y numerosas fotocopias. Durante las visitas de archivo, y posteriormente, había organizado los materiales sobre las aldeas en archivos separados que - me enorgullece decirlo- eran formidables carpetas gruesas. ${ }^{5}$ Mientras me preparaba para escribir el artículo, cuando buscaba estos materiales encontré la carpeta sobre Bisrampur con bastante facilidad, pero faltaba la referente a Balodgahan. Después de varias semanas y muchas búsquedas la paciencia se convirtió en pánico. Revisé mis pilas de documentos una y otra vez y nada más parecía estar perdido, salvo esa carpeta que había desaparecido.

¿Qué podía explicar el misterio de los materiales faltantes? $\mathrm{Al}$ reconstruir mis pasos volvía a un escenario único en repeti-

${ }^{4}$ Para discusiones más extensas sobre los embrollos evangélicos en la India central véase, por ejemplo, Saurabh Dube, Stitches on Time: Colonial Textures and Postcolonial Tangles, Durham, Duke University Press, 2004, especialmente los capítulos 1 y 2. Véase también Saurabh Dube, Untouchable Pasts: Religion, Identity, and Power among a Central Indian Community, 1780-1950, Albany, State University of New York Press, 1998.

${ }^{5}$ Los materiales sobre Bisrampur se encuentran en la Eden Archives and Library en Webster Groves, Misuri, mientras que aquellos sobre Balodgahan son parte de las colecciones de los Archives of the American Mennonite Church en Goshen, Indiana. Trabajé en estos archivos durante varios meses en la década de 1990. 
das ocasiones. En la segunda mitad de los noventa, durante un viaje de trabajo de campo a Chhattisgarh había llevado algunos documentos de archivo que me ayudarían a seguir la pista de personajes clave y episodios importantes en el desentrañamiento de los enredos evangélicos, para establecer una interacción entre el pasado y el presente. Los documentos incluidos en la carpeta sobre la aldea de Balodgahan contienen detalles sobre acuerdos referentes a la tenencia de la tierra y las propiedades en la aldea, así como notas sobre sus habitantes en los primeros años del siglo $\mathrm{XX}$.

Durante mi estancia cerca de Balodgahan, algunas personas se enteraron de que yo poseía tales materiales y unos cuantos manifestaron interés en ellos. ¿Había sido extraída la carpeta de mi habitación, que con frecuencia permanecía abierta y a la cual se podía acceder fácilmente? ¿Se dio el caso, quizá, de que alguien que posiblemente tenía mayores derechos a ella la haya tomado? No estoy señalando a nadie; después de todo, por improbable que parezca, bien pude haber extraviado la carpeta, ya sea en el campo o en casa. Más bien sugiero que, aunque nadie la haya tomado de mi habitación a propósito, la atención y la curiosidad que había por los documentos de Balodgahan atestiguan, a su manera, la continua importancia de las aldeas en la India contemporánea. Los pasados en curso de la aldea resuenan en el presente que se desvanece.

Además de la moraleja de la historia, los materiales faltantes implican que mi ensayo tiene que ser diferente del que originalmente había planeado; de hecho, lo que ofrezco no es un ensayo en el sentido estricto de la palabra, más bien lo que planteo a veces se denomina "artículo de fondo", pero yo preferiría describirlo como una unión de retazos. Tenemos aquí una labor de unión de retazos que se inicia hilvanando y a la vez desentrañando patrones de la aldea en algunos de los tejidos históricos y etnográficos de la India del siglo xx.

Esta labor consta de tres partes, cada una entretejida con la otra de manera que las tres se superponen y al mismo tiempo se extienden una sobre la otra. Para comenzar, plantearé ciertas cuestiones críticas sobre el pasado y el presente de la aldea en la antropología de India, preguntas que establecen el tono para los siguientes pasos; a continuación subrayaré el lugar de 
la aldea en procesos de engranaje evangélico, y por último, abordaré la preponderancia de la aldea en los involucramientos entre la ley colonial moderna y las legalidades populares contemporáneas. En conjunto, tomando como base aspectos de mis escritos anteriores y de mi investigación en curso, la intención es explorar la aldea expresada e ilustrativa a la vez de diseños amplios pero de texturas íntimas de conocimiento antropológico y configuraciones disciplinarias, de imperio y modernidad, cristianismo y conversión, y estado y Nación.

Todo ello se vincula con otro objetivo más de la labor de unión de retazos. Dicho propósito es amplio y franco, y de hecho está motivado críticamente a su propia manera. Registrar la forma en la que los materiales, los objetos y los temas de investigación -en este caso, documentos para el estudio de las aldeas, pero también las aldeas mismas, especialmente sus habitantes y aquellos que las estudian (o estudian en ellas) - se encuentran, se pierden y se recuperan; la unión de retazos revela asimismo la contingencia que marca los emprendimientos académicos. Enfrentar tal contingencia es abandonar las presunciones de una academia hipermuscular que se hace pasar por ciencia desinteresada, que siempre sabe dónde ha iniciado y ya sabe cómo terminará. En lugar de ello, se trata de sondear prudentemente la interpretación académica cambiante de los objetos, los temas y la investigación, sin dejar al último nuestros propios esfuerzos.

No resulta sorprendente que en el trabajo de unión de retazos que seguirá yo reconozca y exprese los múltiples mapeos y las agudas ironías que han caracterizado a las aldeas como parte de diseños más amplios de autoridad, de aprehensión y de alteridad, y también como elementos constituyentes de detalles específicos de la práctica, el proceso y el poder. Resulta lógico que asuma estas tareas mientras abordo y exploro la aldea no simplemente como un objeto de conocimiento sino también como una condición del conocimiento, ya sea al plantear preguntas sobre el lugar de los estudios de la aldea en la disciplina antropológica o al desentrañar las implicaciones de la aldea con el proyecto misionero y un cristianismo vernáculo, o al seguir el rastro de la presencia de la aldea en la dinámica de la ley estatal moderna y las legalidades populares contemporáneas. 
Por supuesto, he estado pensando y escribiendo sobre estos temas en un sentido similar durante algún tiempo ya, pero la expresión particular que mi investigación y mi reflexión encuentran en el presente no habría sido posible si mi mano y mi mente no se hubieran visto forzadas por la pérdida contingente de los materiales de Balodgahan. Hay muchas maneras de encontrar, perder y recuperar las aldeas.

\section{Asuntos formativos}

No sólo la ironía, sino también la ambivalencia rodean al estatus de la aldea en la antropología-sociología del subcontinente. Hoy, los estudios de la aldea suelen abordarse como un paradigma dominante en los entendimientos etnográficos de India tras la independencia, antes de que fueran reemplazados firmemente por una práctica disciplinaria más profética a partir de mediados de la década de $1960 .{ }^{6} \mathrm{Al}$ mismo tiempo, considérese que el socavamiento de la aldea como punto focal de la investigación etnográfica tiene una historia más prolongada de lo que aparenta en un inicio. Dicho cambio estuvo implícito, por ejemplo, en las propuestas de Louis Dumont para la "sociología de India”. ' Además de los muy discutidos énfasis en la expresión de la disciplina ubicada en la coyuntura de la indología y la etnografía, se observaba una agenda centrada principalmente en la casta y el parentesco como los elementos básicos de la sociología-antropología. En este sentido destacan dos puntos: ${ }^{8}$ por un lado, en línea y a la vez en oposición con Dumont, algunas expresiones importantes de la antropología-sociología del subcontinente se alejaron de preocupaciones clave de los estudios de la aldea; por otro lado, resulta igualmente pertinente que en corrientes significativas de tal emprendimiento, la aldea

${ }^{6}$ Para una discusión más amplia de tales cuestiones, véase Saurabh Dube, “Anthropology, History, Historical Anthropology”, en Saurabh Dube (ed.), Historical Anthropology, Nueva Delhi, Oxford University Press, 2007, pp. 1-73.

7 Véase particularmente Louis Dumont y David F. Pocock, "For a Sociology of India”, Contributions to Indian Sociology, vol. 1, 1957, pp. 7-22.

${ }^{8}$ Algunas consideraciones de los contextos académicos más amplios que dan forma a estos sucesos aparecen en Dube, "Anthropology, History, Historical Anthropology", op. cit. 
con frecuencia permaneciera como el locus de la investigación, pero no su problemática central. Es decir, mientras los estudios clásicos de la aldea habían abordado diversos temas tomando la aldea como su rúbrica organizacional y su preocupación focal, el cambio en tales estudios académicos bien podría depender de utilizar la aldea principalmente como un entorno en el cual llevar a cabo investigaciones discretas. ${ }^{9}$ Considerando todo, $\mathrm{mi}$ argumento implica reconocer los requisitos de prestar una atención cuidadosa a los múltiples mapeos de la categoría-entidad de la aldea en la antropología de India.

La multiplicidad misma menoscaba suposiciones presentes cargadas, generalmente a priori, sobre los "estudios de la aldea" como modalidades de conocimiento anticuadas y homogéneas, en las que la aldea y el mundo encontraron expresiones exclusivas. Resulta crítico reexaminar no sólo esas presuposiciones actuales, sino también el o los conocimientos pasados que conjuran. Debería ser suficiente plantear tres preguntas: a pesar de suposiciones disciplinarias presentes en el sentido de que las consideraciones sobre la transformación de la sociedad simplemente estaban ausentes de los estudios clásicos de la aldea, ¿quizás los procesos históricos encontraron una expresión ambigua en tal emprendimiento académico, lo que incluye trabajos basados en premisas funcionalistas? ¿Algunos de estos escritos anticiparon de manera ambivalente desviaciones críticas en la etnografía contemporánea? ¿Acaso no es cierto que la ambigüedad y la ambivalencia siguen caracterizando hoy las concepciones sobre la temporalidad y la modernidad en la antropología en general?

Al abordar dichas preguntas tomo un ejemplo específico, el del innovador estudio del antropólogo S. C. Dube, Indian Village. ${ }^{10}$ Espero que estos ejemplos sirvan para asentar los temas que he estado planteando. ${ }^{11}$ Indian Village fue un estudio de

${ }^{9}$ Sobre tales lineamientos, véase Mines y Yazgi (eds.), Village Matters, op. cit. Véase también la nota 16 , más adelante.

${ }^{10}$ S. C. Dube, Indian Village, Londres, Routledge and Kegan Paul, 1955. Véase también S. C. Dube, India's Changing Villages: Human Factors in Community Development, Londres, Routledge and Kegan Paul, 1958.

${ }^{11}$ Esta discusión se basa en mi ensayo detallado sobre la vida y el trabajo de Dube. Saurabh Dube, “Ties that Bind: Tribe, Village, Nation, and S. C. Dube”, en Patricia Uberoi, Satish Deshpande y Nandini Sundar (eds.), Disciplinary Biographies: 
Shamirpet, que se encuentra en la región de Telangana, a 40 kilómetros de Hyderabad, ciudad en la que Dube había empezado a dictar cátedra, en la Universidad de Osmania. La investigación para ese libro surgió de un proyecto de equipo bastante amplio en esta institución a inicios de la década de 1950. El objetivo principal del proyecto fue el bienestar de la aldea y la investigación rural como parte de la construcción de la nación en lo que había sido un Estado principesco. La elección de Shamirpet implicó consideraciones tanto prácticas como sociológicas. Shamirpet no le quedaba demasiado lejos a los miembros del proyecto para poder ir y volver desde Hyderabad el mismo día, y además en términos de su población, área y composición de castas era "representativa" de las aldeas de la región.

Las investigaciones antropológicas del proyecto se centraban en temas de la estructura social, económica, ritual, de parentesco y familiar de la aldea. Además de un censo sociológico general, las indagatorias implicaron investigaciones intensivas a través de una muestra de 120 de un total de 380 familias; 80 historias de vida episódicas y tópicas y once biografías completas registradas a través de entrevistas por asociación libre, así como el uso de observación participante y el estudio de registros sobre la aldea que se encontraban disponibles. La investigación que llevó a cabo el proyecto Osmania en la aldea de Shamirpet fue muy amplia en su alcance y profundidad. Al final del año, Dube tenía tres mil páginas de notas mecanografiadas y clasificadas, que conformarían la base de Indian Village. Dube se sumergió en las notas y en los datos, realizó un esbozo y redactó el borrador de dos capítulos en el verano de 1952, antes de terminar el estudio a lo largo del siguiente año como catedrático visitante en la School of Oriental and African Studies en Londres.

Indian Village se realizó con un estilo simple y una redacción elegante que en conjunto tuvieron como resultado un retrato vívido y atractivo de la aldea. Al mismo tiempo, las técnicas narrativas del libro se vincularon a sus términos de teo-

Essays in the History of Indian Sociology and Anthropology, Delhi, Permanent Black, 2007, pp. 444-495. 
ría; en este sentido, un funcionalismo latente se entretejió en la textura del recuento sin obstruirla, y la presentación de normas dominantes y sus principales variaciones, donde la "personalidad" suele "explicar" las últimas divergencias, apuntaló la descripción. Una terminología estrecha se entrelazó con la narración, y las categorías analíticas y los materiales empíricos se enredaron los unos con los otros. No resulta sorprendente que en una reseña no firmada en The Times, de Londres, el maestro Edmund Leach alabó el libro no sólo por su excelencia descriptiva, sino también por la riqueza misma de los materiales que presentó, que plantearon nuevos cuestionamientos a la teoría antropológica.

Cincuenta años después, la evaluación de Leach puede abordarse y ampliarse de diferentes maneras. En Indian Village, la prosa fluida de Dube, que une perfectamente lo analítico y lo empírico, contiene tendencias contrarias que se encuentran en el corazón de la narrativa. Considérese aquí la tensión entre el pasado y el presente, las cargas de la historia y las demandas de la antropología, en el núcleo de la obra. Dube comenzó por cuestionar la noción de una aldea totalmente "representativa", al plantear los temas en términos de las importantes distinciones y las similitudes estructurales entre las aldeas en India. Argumentó que "no podemos ver a la comunidad aldeana india como estática, atemporal e inmutable. El tiempo y la interacción de factores y fuerzas históricas y sociológicas han influido en la estructura, la organización y el etos de estas comunidades de muchas maneras significativas". ${ }^{12}$ No obstante, en la narrativa, las transformaciones a través del tiempo aparecieron principalmente al inicio y al final del recuento. En este sentido, los primeros pasos fueron los mecanismos de encuadre, mientras que los últimos conformaron el final magistral, que asumió la forma de un capítulo integral donde se describen los cambios en la aldea, en el pasado y en el presente.

Sugiero, entonces, que términos como historia, cambio y transformación se encuentran presentes en Indian Village: por un lado, su labor habita los extremos del recuento e insinúa sus fines, con lo que marca una brecha entre el cambio y la trans-

${ }^{12}$ Dube, Indian Village, op. cit., pp. 3-7. 
formación que provienen de fuera de la aldea, y la continuidad y la estabilidad inherentes a ella. Esto establece una división entre la historia externa y la estructura interna; por otro lado, la narrativa presenta igualmente procesos históricos y desarrollos contemporáneos que abarcan la aldea, lo que inserta e instituye a Shamirpet en un presente etnográfico perdurable. Esto marca descriptivamente a las aldeas como un lugar en la historia, pero analíticamente como una entidad fuera del tiempo. Más que estar simplemente sobredeterminados por planos teóricos, los atributos gemelos son resultado de la estructura del trabajo y de su estilo de escritura, que actúan en conjunto. ${ }^{13}$ Asimismo, en lugar de abordar las tensiones que se aprecian en toda la obra como meramente incapacitantes, es importante monitorearlas como vertientes en oposición que constituyeron una antropología-sociología emergente de la India independiente, un estudio académico moldeado a la sombra del imperio y a la luz de la nación.

Todo esto tiene amplias implicaciones, pues es importante no sólo reiterar los requisitos de evaluaciones más cuidadosas sobre la vida de la aldea en los estudios académicos del subcontinente, sino también registrar la falta de historias críticas de la antropología-sociología del sur de Asia. ${ }^{14}$ En tal escenario, permítaseme subrayar otras tres cuestiones que, en conjunto, apuntan hacia tareas críticas futuras y además sugieren posibles formas de entender por qué la aldea sigue siendo un elemento infaltable en la enseñanza y la investigación en varias universidades provinciales y algunas metropolitanas en India hoy en día.

${ }^{13}$ Considérense juntas la organización de la primera monografía de Dube, The Kamar, y la de Indian Village, que siguió apenas unos años después. Ambas obras muestran un parecido familiar y discuten los cambios que afectaron los temas estudiados principalmente al final de sus recuentos. Al mismo tiempo, en The Kamar, a pesar de sus predilecciones más amplias sobre una estructura social inmóvil, Dube no pudo refrenar las transformaciones de la "tradición" entre los pueblos kamar, que eran cazadores-recolectores, no sólo al final sino incluso en el corazón del estudio. En contraste, la escritura lograda y elegante de Dube en Indian Village logró integrar tales tensiones de manera más prolija en el flujo de la narrativa. S. C. Dube, The Kamar, Lucknow, Universal, 1951.

${ }^{14}$ Ello no implica que niegue la existencia de notas orientadas a tal esfuerzo, tema que discuto (junto con su importancia) en "Anthropology, History, Historical Anthropology", op. cit. 
Primero, es necesario retomar algunas discusiones destacadas sobre negaciones persistentes de la temporalidad a objetos de investigación antropológica en el contexto del estudio etnográfico de la sociedad india, ${ }^{15}$ cuanto más si nos centramos en estudios de la aldea después de la independencia, así como en antropólogos del subcontinente que estudian sociedades del sur de Asia, en términos más generales. Aquí se encuentran cuestiones de construcciones simultáneas y contrarias en la etnografía, que abarcan objetos atemporales de suposición antropológica que también fueron temas contemporáneos en el tiempo y el espacio de la nación. ${ }^{16}$ Por lo tanto, atender estos temas implica estar alerta a dos procesos sobrepuestos: la manera en que la teoría metropolitana llegó a traducirse en la práctica, a través de sus aplicaciones en el contexto académico de una nueva nación, y la forma por la cual los procedimientos

${ }^{15}$ Las cuestiones sobre tal escisión de la temporalidad encuentran expresión en discusiones sobre la "negación de la contemporaneidad" entre el antropólogo autor y el objeto antropológico (Johannes Fabian), sobre las "tierras de nunca jamás” de la etnografía (Bernard Cohn), sobre el "sitio del salvaje" en la antropología (Michel-Rolph Trouillot) y sobre la persistencia de "espacios encantados y sitios modernos" (Saurabh Dube) y de lo "exótico en casa" (Micaela di Leonardo) en los mundos académicos y sociales. Johannes Fabian, Time and the Other: How Anthropology makes its Object, Nueva York, Columbia University Press, 1983; Bernard Cohn, "History and Anthropology: The State of Play", Comparative Studies in Society and History, vol. 22, núm. 2, 1980, p. 199; Michel-Rolph Trouillot, "Anthropology and the Savage Slot: The Poetics and Politics of the Otherness", en Richard Fox (ed.), Recapturing Anthropology: Working in the Present, Santa Fe, School of American Research Press, pp. 17-44; Dube, Stitches on Time, op. cit., y Micaela di Leonardo, Exotics at Home: Anthropologies, Others, and American Modernity, Chicago, University of Chicago Press, 2000. Véase también John Comaroff y Jean Comaroff, Ethnography and the Historical Imagination, Boulder, Westview, 1992, pp. 1-9; Nicholas Thomas, Out of Time: History and Evolution in Anthropological Discourse, Ann Arbor, University of Michigan Press, 1996; Catherine Lutz y Jane Collins, Reading National Geographic, Chicago, University of Chicago Press, 1993, y Dube, “Anthropology, History, Historical Anthropology”, op. cit.

${ }^{16}$ El tema sólo se extiende a otras obras antropológicas sobre el sur de Asia, como aquellas que se publicaron aproximadamente en la época del libro de Dube, incluidas colecciones de estudios de la aldea editadas por M. N. Srinivas y McKim Marriott, así como el estudio seminal de Srinivas sobre los kodava hindúes (que, no obstante, anteriormente había tenido una encarnación diferente bajo la supervisión de G. S. Ghurye en la década de 1940). M. N. Srinivas (ed.), India's Villages, Bombay, Media Promoters and Publishers, 1955; McKim Marriott (ed.), Village India: Studies in the Little Community, Chicago, University of Chicago Press, 1955, y M. N. Srinivas, Religion and Society among the Coorgs of South India, Oxford, Clarendon Press, 1952. Considérese también, M. N. Srinivas, Social Change in Modern India, Berkeley, University of California Press, 1966. 
indicados reflejan las tensiones contradictorias que podrían apuntalar la antropología metropolitana.

En segundo lugar, para analizar el pasado y el presente de la aldea en el estudio académico de India, resulta crucial entablar otras exploraciones críticas. Por un lado, la forma en que los escritos en la antropología-sociología del subcontinente, a partir de la década de 1950, llegaron a formarse con moldes cada vez más sincrónicos, particularmente bajo la influencia de análisis funcionalistas, estructurales-funcionalistas y estructurales. Así, tales medidas colocan un signo de interrogación en lo que ahora aparece como los procedimientos especulativos y las proyecciones de sentido común - sobre todo en lo concerniente a la historia, la civilización y la tradición- en el estudio académico anterior de estas disciplinas. ${ }^{17}$ Por el otro lado, los protocolos particulares por medio de los cuales las consideraciones de los términos de la civilización y la tradición indias, incluidas expresiones con frecuencia implícitas de patrones de la historia, no desaparecieron simplemente de los escritos más recientes, sino que se doblaron de diversas maneras en los pliegues de los análisis científicos (sociales) mejor organizados, estructurados más sincrónicamente y con mayor seguridad; en

${ }^{17}$ En otros textos he mostrado que la obra de importantes antropólogos y sociólogos indios, cuya reputación ya estaba bien establecida antes de la independencia, con frecuencia se vio moldeada por presuposiciones implícitas y proyecciones explícitas sobre la civilización y la historia del subcontinente. Esto sucedió con académicos tan diversos como Nirmal Kumar Bose, G. S. Ghurye, Iravati Karve, Radhakamal Mukherjee, D. P. Mukerjee y D. N. Majumdar. Fueron los escritos de tales antropólogos y sociólogos los que llegaron a ponerse en entredicho, a partir de la década de 1950, a través de procedimientos académicos que, sugiero, requieren un entendimiento prudente. De hecho, sería apresurado simplemente aceptar los términos de tal cuestionamiento de manera que los entendimientos académicos previos se prejuzguen como conocimiento anticuado, que se volvió obsoleto después de los años cincuenta por una práctica cada vez más especializada de la antropología-sociología de India. En lugar de ello, una tarea más difícil pero más productiva implica rastrear las maneras específicas y cambiantes en que se agotaron las nociones y los entendimientos de la tradición, la historia y la civilización en la obra de académicos que adquirieron prominencia antes y después de la independencia. Dube, "Anthropology, History, Historical Anthropology”, op. cit. Véase también T. N. Madan, Pathways: Approaches to the Study of Society in India, Delhi, Oxford University Press, 1994, pp. 3-36, y Ravindra K. Jain, "Social Anthropology of India: Theory and Method", en Indian Council of Social Science Research, Survey of Research in Sociology and Social Anthropology, 1969-1979, Nueva Delhi, Satvahan, 1985, pp. 1-50. 
particular, los estudios de la aldea. ${ }^{18}$ En conjunto, emprender estos esfuerzos sería desentrañar una vez más los lineamientos de los archivos de la antropología india, sobre todo sus implicaciones con el imperio y la nación, las categorías occidentales y las consideraciones nacionalistas, y sus expresiones. Nada de ello sugiere una simple celebración de tales estudios; más bien implica los requerimientos de entrar en los protocolos de este conocimiento y de sus formas de conocer de manera crítica, pero cautelosa, con el fin de analizar sus consideraciones $y$ contradicciones constitutivas, sus ambivalencias y sus excesos formativos.

En tercer y último lugar, abordar los temas que recién se esbozaron -incluidos los que involucran expresiones de la civilización, la tradición y la historia indias- como recuentos influyentes que dan forma y suturan, relativos a la aldea y la estructura social del subcontinente en la antropología después de la independencia, exige otra tarea. Se trata de la tarea inconclusa de comprender el legado que dejó a la antropología-sociología de India a lo largo del siglo xx, la presencia anterior, la tradición extensa, de los escritos coloniales y nacionalistas sobre la aldea en el sur de Asia. ${ }^{19}$ Tales preocupaciones adquieren una mayor fuerza a la luz de los argumentos que sostienen que las formas coloniales de conocimiento-poder pasaron de ser modalidades "históricas" en la primera mitad del siglo XIX a "etnográficas" durante el último siglo de dominio imperial. ${ }^{20}$

Si la aldea parece haberse caído del mapa en la mayoría de los emprendimientos antropológicos críticos (y no tan críticos) de los últimos años, esta aparente desaparición también ha

${ }^{18}$ Las formulaciones de Robert Redfield sobre las "grandes” y las "pequeñas" tradiciones de las civilizaciones ejercieron una importante influencia en este sentido. McKim Marriott pudo preguntar lo siguiente, al tiempo que expresaba esta influencia: “PPuede una aldea comprenderse y concebirse como un todo por sí misma, y entender una aldea como tal puede contribuir a comprender la cultura más amplia [...] en la que se arraiga la aldea?". Robert Redfield, Peasant, Society, and Culture, Chicago, University of Chicago Press, 1956; Marriott (ed.), Village India, op. cit., p. 171. Véase también Jain, "Social Anthropology of India", op. cit., pp. 8-11, y Satish Saberwal, "Sociologists and Inequality in India: The Historical Context", Economic and Political Weekly, vol. 14, núm. 7-8, 1979, pp. 243-254.

${ }_{19}$ Véase, por ejemplo, Ronald Inden, Imagining India, Oxford, Basil Blackwell, 1990, especialmente las pp. 131-151.

${ }^{20}$ Dirks, Castes of Mind, op. cit. 
implicado ambivalencias relacionadas. Por un lado, como es bien sabido, en la etnografía y la antropología histórica más recientes, un cuestionamiento cercano de los límites de lo "local" se ha acompañado por la esquematización plural de sociedades y culturas. Las explicitaciones de la práctica, el proceso y el poder - que con frecuencia se proyectan como una vanguardista trinidad analítica de la antropología crítica- y los énfasis en procedimientos transnacionales, culturas que se intersecan $\mathrm{e}$ historias sobrepuestas han implicado que la aldea no ha tenido demasiada suerte aquí, pues ha llevado el estigma de ser, bueno, demasiado "local". ${ }^{21}$ Si bien el propósito del presente ensayo es precisamente cuestionar tales proyecciones, sería ridículo no aprender de las tensiones del estudio académico crítico que se discuten. Cuando menos señalan la salida del subcontinente indio como un fin antropológico en sí mismo. Por otro lado, el aprendizaje exacto en este sentido debe acompañarse por un cuestionamiento prudente de las suposiciones formativas de varias corrientes de la antropología nueva e influyente. Considérense, por ejemplo, los pronunciamientos programáticos en favor de una etnografía multisituada o de una antropología novel para un mundo cambiante. ${ }^{22}$ Dejemos a un lado, por el momento, el hecho de que el mundo ha estado cambiando durante mucho tiempo. Mi preocupación tiene que ver con la manera en que tales llamamientos se han asimilado fácilmente a desechos simplistas de tradiciones antropológicas por ser completamente defectuosos y cómplices con el poder institucional. Como resultado, en los últimos 15 años una cierta clase de sabiduría etnográfica ha compartido la presunción contemporánea que "exagera la calidad única de nuestro tiempo". ${ }^{23}$ Tal

${ }^{21}$ Sobre estos desarrollos académicos más amplios, véase Dube, “Anthropology, History, Historical Anthropology”, op. cit., y Dube, Stitches on Time, op. cit.

${ }^{22}$ Más allá de académicos específicos y escritos particulares, tales pronunciamientos y presuposiciones estaban en boga en la década de 1990 y a inicios de la de 2000 , y se exhibían con prominencia en influyentes departamentos de antropología y organismos que otorgaban becas en Norteamérica. Es decir, fueron la ortodoxia crítica de la antropología cultural, una clase de sentido común tácito, más prevalente que meros productos y practicantes intelectuales.

${ }^{23}$ Michel-Rolph Trouillot, "North Atlantic Universals: Analytical Fictions, 1492-1945”, en Saurabh Dube (ed.), Enduring Enchantments, edición especial de South Atlantic Quarterly, vol. 101, núm. 4, 2002, p. 840. 
suposición también legisla singularmente sobre la relevancia de la investigación antropológica y considera que se basa en la supuesta novedad del tema de investigación. El punto aquí es que hay problemas con las presuposiciones que (con frecuencia, implícitamente) se apoderan de objetos aparentes de investigación intelectual, para establecerlos como el criterio exclusivo para juzgar el punto de partida académico y la validez teórica del emprendimiento intelectual.

En términos concretos, sugiero que no hay nada intrínseco en, digamos, los recuentos etnográficos multisituados del art déco -o, para tal caso, el baile a gogó- en Shanghái, en la Ciudad de México y en Nueva York, que los hagan más atractivos y relevantes que un estudio crítico de la casta y el parentesco en las aldeas indias, por ejemplo. Más allá del alud de requisitos para la investigación y de la charla académica, en cada caso, mucho depende de las preguntas que se planteen, las verdades que se desaprendan, los conceptos que se invoquen, las realidades que se deshagan, las categorías que se evoquen y las narrativas que se traigan a escena. ¿Acaso no son las preguntas y los conceptos - y el desaprendizaje de las verdades- los que dan forma a los objetos de investigación, especialmente al desentrañar (o ignorar) las relaciones en las que se arraigan? ¿Acaso no son las categorías y las narrativas -y la anulación de las verdades- las que pueden llevar a los objetos (de una razón única) que se interpretan como sujetos (con diferentes razones)?24 ¿Pueden no tenerse en cuenta aquí la práctica, el proceso y el poder? Está en juego no sólo el cuestionamiento de presuposiciones prevalentes sobre la aldea, sino también la afirmación crítica y cautelosa de la categoría-entidad; de hecho, es en este sentido que hablo de la aldea no meramente como un objeto de conocimiento, sino como una condición del conocer.

${ }^{24}$ Discuto tales cuestiones a través de un recuento etnográfico de los ámbitos académicos en After Conversion: Cultural Histories of Modern India, Nueva Delhi, Oxford University Press, 2010. 


\section{Cultivos y rebaños}

La aldea india fue sumamente importante para el proyecto de la misión, ${ }^{25}$ pues fue en ella donde los misioneros pusieron sus esperanzas milenarias; fue ahí donde aprendieron por primera vez sobre la conversión en el terreno; fue en la aldea donde los misioneros entretejieron fácilmente, pero sin descanso, procedimientos de proselitismo evangélico con protocolos de la civilización occidental. Por supuesto, la casualidad ejerció cierta influencia en el establecimiento de estaciones misioneras y aldeas cristianas. Cuando el misionero Oscar Lohr llegó a Raipur, en 1868, encontró a un benefactor y un aliado en el coronel Balmain, el comisionado en jefe de la región de Chhattisgarh. El funcionario aconsejó al misionero que adquiriera un lugar para establecer una estación misionera, y le informó que un gran terreno baldío, propiedad del gobierno, con una extensión de 625 hectáreas, estaba a punto de ser subastado públicamente. Esto encajaba perfectamente con los planes de Lohr de comenzar su trabajo en "terrenos indómitos", entre las castas bajas. Con ayuda financiera del coronel Balmain, Lohr compró el terreno y lo llamó Bisrampur, morada de descanso; sin embargo, la aldea también era el lugar de trabajo del evangelista. Al cabo de algunos meses, la familia de Lohr se había mudado a un búngalo con letrinas. El terreno incluía una aldea desierta llamada Ganeshpur. Como parte del acuerdo con los malguzares (propietarios de aldeas) que inició el régimen colo-

${ }^{25}$ Los temas que se plantean en esta sección se derivan de mi estudio, más extenso, de las implicaciones evangélicas, a horcajadas entre los pasados de la India central y las historias norteamericanas, en los siglos XIX y XX. El proyecto en curso monitorea la ubicación contradictoria de la empresa misionera en la creación de culturas coloniales; rastrea el entrelazamiento de prácticas protestantes con dinámicas de casta-secta en la creación de un cristianismo vernáculo, y explora expresiones variadas de comunidad y nación tras la conversión. El proyecto se basa en trabajo de archivo en colecciones misioneras relativas a India central y Norteamérica, y en trabajo de campo en India central y Estados Unidos. Esta labor ha implicado más de un año de investigación de archivo en registros de misioneros y colecciones históricas pertenecientes a la Sociedad Misionera Evangélica Alemana-Americana (St. Louis y Cambridge, Massachusetts), la Misión contra la Lepra (Londres), los Menonitas de la Conferencia General (Newton, Kansas), la Iglesia Menonita Estadounidense (Goshen, Indiana), los Discípulos de Cristo (Nashville) y los Metodistas (Madison, Nueva Jersey). Además, se ha acompañado de varios meses de trabajo de campo en la zona central de India y el medio oeste de Estados Unidos. 
nial en la década de 1860, el misionero quedó registrado como malguzar de Bisrampur y Ganeshpur. Desde el principio, el poder temporal del misionero llegó a radicar en derechos de propiedad que se extendían al bosque en Bisrampur y Ganeshpur; asimismo, el misionero era el líder espiritual de la aldea cristiana y la estación misionera. ${ }^{26}$

Lohr había establecido un precedente. A comienzos del siglo Xx, poco después de que los evangelistas de la Misión Menonita Americana hicieran del pueblo de Dhamtari su base de operaciones, compraron la aldea de Balodgahan. El misionero A. C. Brunk describió la adquisición de la siguiente manera:

Dado que la agricultura era la principal ocupación de la mayoría de la gente de India, resultaba natural que el misionero pensara que la labranza era la actividad preponderante de un gran número de cristianos. Teniendo esto en cuenta, se hizo un esfuerzo por comprar una aldea con dicho propósito. Después de mucha investigación [...] se eligió a Balodgahan [...] La aldea se encontraba a siete millas al suroeste de Dhamtari. Balodgahan fue comprado a Appajee a Mahrata [Maratha] por un monto de 2700 u 8000 rupias. $^{27}$

El elemento un tanto "secular" que evoca esta descripción era congruente con el hecho de que, a diferencia de Bisrampur, Balodgahan nunca fue una comunidad puramente cristiana; más bien, la intención era que se convirtiera en una aldea económica modelo. Aquí también el misionero era tanto el malguzar como el pastor, pero los derechos de propiedad de la misión se extendían únicamente a 60 hectáreas de la extensión total de Balodgahan, que eran 335 hectáreas. De esas 60 hectáreas, 42 se destinaron a pastoreo. ${ }^{28} \mathrm{~A}$ lo largo de los años, los terrenos que poseía la misión eran cultivados por aparceros o simplemente se rentaban por sumas en efectivo. En el segundo caso, la misión podía apartarle a una casa para viudas o un orfanatorio de niñas las parcelas que ellas desearan cultivar y recibía a cambio una proporción fija de los productos de la siembra. En lugar de desalojar a los agricultores de castas medias y bajas y a

${ }^{26}$ Para mayores detalles, véase Dube, Untouchable Pasts, op. cit., pp. 70-73.

27 A. C. Brunk, "Balodgahan Village", manuscrito IV-17-21, caja 28, Charitable Institutions, f. 1, Goshen, Archives of the American Mennonite Church, sin fecha.

${ }^{28}$ Idem. 
los adivasi, que representaban a la gran mayoría en Balodgahan, la misión menonita siguió la política de comprarles terrenos cuando los ponían a la venta y después vendérselos a miembros de la comunidad cristiana. Para finales de la década de 1920 había alrededor de 30 agricultores cristianos en la aldea. ${ }^{29}$ Todo ello contrastaba con Bisrampur, donde cada hogar cristiano originalmente había recibido de la misión 1.6 hectáreas de tierra y donde (también a finales de los veinte) un gran número de los 183 hogares tenían alguna relación con la agricultura. ${ }^{30}$ De hecho, Bisrampur y Balodgahan revelan texturas y vínculos de paternalismo definidos - así como de disciplina misionera- que espero explorar con mayor detalle en otros textos. Después de todo, el cultivo y el pastoreo de rebaños están marcados por densas historias.

Resulta interesante comparar a Bisrampur con Balodgahan y analizar sus vínculos. Un ejemplo son los entendimientos de los evangelistas sobre la conversión. Por supuesto, había diferencias entre el milenarismo de mediados del siglo XIX de Lohr y el evangelismo del siglo xx de los menonitas. No obstante, yo sugeriría que una cierta transformación milagrosa en el horizonte animó, de manera intermitente pero inexorable, todas las expresiones evangélicas en el "desierto espiritual" de la India central. Si Lohr tuvo sus propios trazos cartográficos de la transformación, en el siglo xx el tropo del "movimiento masivo" gozó de un favor especial entre los misioneros. No resulta sorprendente que el locus primario requerido para tales transformaciones fuera la India aldeana, que era la ciudadela de la casta. ${ }^{31}$

La empresa evangélica adquirió forma por la interacción entre concepciones opuestas pero sobrepuestas de la conversión. En primer lugar, los misioneros llevaron la carga de las proyecciones paulinas sobre la conversión, vinculadas a diversas visiones de un solitario Saúl viendo la luz, elemento que lle-

\footnotetext{
${ }^{29}$ Idem.

${ }^{30}$ [Reverendo] Miller, "Survey of Bisrampur", Bisrampur Malguzari File, Webster Groves, Eden Archives and Library, 1925.

${ }^{31}$ Ello se pone de manifiesto en los materiales de archivo -como la correspondencia (oficial y personal), los diarios y los reportes (anuales, trimestrales y mensuales) - que generaron, a partir de la década de 1920, los evangelistas de todas las organizaciones misioneras ya mencionadas.
} 
varon consigo desde su pasado en Estados Unidos y sigue vivo en su presente en India. ${ }^{32}$ En segundo lugar, aspecto también vinculado a la vida del Libro, los milagrosos poderes del Señor para sanar a los enfermos siguieron figurando prominentemente en los recuentos misioneros como elemento que convencía a las personas a adoptar el cristianismo. ${ }^{33}$ En tercer y último lugar, los evangelistas elaboraron de diversas maneras el tema de los vínculos "naturales" de parentesco y casta como pilar para transformaciones de la fe variadas y colectivas. ${ }^{34}$ El entrelazamiento y las tensiones entre estos conceptos sobre la conversión estuvieron en el centro de la empresa evangélica en Bisrampur y en Balodgahan, y encontraron configuraciones cambiantes y compartidas en ambas aldeas. Tales nudos históricos ponen en entredicho nociones que se asumen automáticamente sobre la conversión y los misioneros en la India contemporánea. ${ }^{35}$

¿Y qué ocurre con la versión de los conversos, su traducción del mensaje misionero y la aldea cristiana en la creación de una modernidad evangélica? ${ }^{36}$ De diferentes maneras, los pasados de Bisrampur y Balodgahan revelan cómo los lazos de paren-

${ }^{32}$ Véase, por ejemplo, Theodore Seybold, "The Reverend Ramnath Simon Bajpai”, texto mecanografiado, 84-9b Bio 52, Eden Archives and Library, Webster Groves, sin fecha. Asimismo, he argumentado que incluso esta exclusiva interpretación de la conversión, a tono con las proyecciones paulinas, se destruyó por la fuerza del mito, la leyenda y la historia, arraigados en procesos más amplios de embrollos evangélicos. Saurabh Dube, "Witnessing Lives: Conversion and Life-history in Colonial Central India”, en Ishita Banerjee-Dube y Saurabh Dube (eds.), Ancient to Modern: Religion, Power, Community, Nueva Delhi, Oxford University Press, 2009, pp. 259-290. Estos énfasis del ensayo se aclaran en textos como los de David Bajpai, "My Father - Rev. Simon Ramnath Bajpai”, texto mecanografiado, 84-9b Bio 52, Webster Groves, Eden Archives and Library, 1945. M. M. Paul, "Autobiography of M. M. Paul, Head Catechist at Mahasamund”, texto mecanografiado, 84-9b Bio 52, Webster Groves, Eden Archives and Library, sin fecha.

${ }^{33}$ Dube, Untouchable Pasts, op. cit., pp. 72-73.

${ }^{34}$ Idem.

${ }^{35}$ Saurabh Dube, "Conversion to Translation: Colonial Registers of a Vernacular Christianity", en Dube (ed.), Enduring Enchantments, op. cit., pp. 807-837; y Dube, "Witnessing Lives", op. cit. Véase también Saurabh Dube e Ishita Banerjee-Dube, "Spectres of Conversion: Transformations of Caste and Sect in India”, en Rowena Robinson y Sathianathan Clarke (eds.), Religious Conversion in India: Modes, Motivations, and Meanings, Nueva Delhi, Oxford University Press, 2003, pp. 222-254.

${ }^{36}$ Sobre tales cuestiones véanse, por ejemplo, mis obras ya citadas: "Conversion to Translation", Stitches on Time, "Witnessing Lives”, y After Conversion. 
tesco y los vínculos de paternalismo demostraron ser críticos para el crecimiento y la consolidación de las congregaciones cristianas en la India central. En ambas aldeas, el misionero era tanto el malguzar como el pastor. En Bisrampur, esto ocultó entre los conversos la división entre la autoridad temporal y la espiritual que profesaban los misioneros; ${ }^{37}$ en Balodgahan, permitió a los habitantes hindúes de la aldea establecer nuevas conexiones entre el ritual y el poder que detentaban los evangelistas. ${ }^{38} \mathrm{Al}$ mismo tiempo, en cada caso, viendo la naturaleza de la autoridad a través de patrones que acentuaban los vínculos indisolubles entre el ritual y el poder, el misionero como pastor y propietario de la aldea podía aparecer a ojos de sus habitantes un raja-log, alguien parecido a un rey. ${ }^{39}$

Todo ello permite una mayor elucidación de la naturaleza de la casta. Anteriormente he argumentado que la pureza-contaminación y la realeza ritual no eran principios opuestos, sino esquemas rituales gemelos de significado y poder. En los siglos XIX y XX, estos esquemas actuaron junto con las formas de dominio derivadas del gobierno colonial para definir patrones de poder en el orden de las castas. ${ }^{40} \mathrm{De}$ modos necesariamente distintos, una investigación de Balodgahan y Bisrampur no sólo sugiere que los esquemas de pureza-contaminación y realeza cultural, en formas modificadas y reelaboradas, podrían moldear los patrones, las prácticas y las percepciones de las congregaciones cristianas, sino que también subraya cómo las continuidades y las rupturas con los términos de casta y secta, incluidos mecanismos de incorporación y ostracismo, adquirieron nuevos sentidos a través de su nueva configuración en comunidades reagrupadas, donde estos procesos implicaron necesariamente transformaciones en las texturas de la vida aldeana y sus manifestaciones institucionales. ${ }^{41}$

${ }^{37}$ Dube, Stitches on Time, op. cit., especialmente el capítulo 2.

${ }^{38}$ Brunk, "Balodgahan Village", op. cit.

${ }^{39}$ De diversas maneras, las descripciones de la pareja misionera como raja-log -además de otras que les llamaban "mamá-papá”- han aparecido en repetidas ocasiones durante mi trabajo de campo en Chhattisgarh.

${ }^{40}$ Dube, Stitches on Time, op. cit., capítulos 2 al 4; Dube, Untouchable Pasts, op. cit.

${ }^{41} \mathrm{Si}$ bien hasta ahora he abordado en mis escritos estas cuestiones sobre el cristianismo, la conversión y el colonialismo, propongo abundar en ellos de manera sostenida en mi proyecto en curso sobre los embrollos evangélicos. 
Tanto en Balodgahan como en Bisrampur, la preocupación de los evangelistas por la monogamia y su miedo al adulterio implicaron que los conversos tuvieran prohibido casarse en segundas nupcias, aunque éstos desobedecieron constantemente la autoridad de los misioneros en este sentido y siguieron celebrando matrimonios secundarios. En la década de 1930, los conversos de Bisrampur se basaron en las prohibiciones misioneras del adulterio y los principios de mantenimiento de los límites entre los grupos, arraigados en las reglas de la casta y la secta, para invocar la amenaza a la castidad de las "hermanas cristianas vírgenes”. Convirtieron el honor de la mujer en una metáfora evocadora de orden en la comunidad y en un símbolo que constituía su límite. Esta comunidad y su límite expresaron una construcción crítica de la aldea. ${ }^{42}$ Aunque sin expresar la aldea de maneras tan categóricas, los conversos de Balodgahan también desafiaron a sus misioneros al dar forma a sus entendimientos de la transgresión sexual. ${ }^{43}$

En cada uno de estos sitios, la crítica y la negociación de la autoridad misionera por parte de los conversos subraya sus usos del cristianismo, representados de diversas maneras a través de la renovación de expresiones evangélicas, de la retórica nacionalista y de los encantos gubernamentales. En otros textos he demostrado que una iniciativa crucial de la congregación de Bisrampur, en la década de 1930, implicó una división profunda entre nosotros y ustedes, entre la comunidad y los fuereños. ${ }^{44}$ Con la comunidad que se formó en torno de los conversos de Bisrampur, todos los empleados que no pertenecían originalmente a la misión fueron considerados "forasteros" y de inmediato se protestó contra su creciente intrusión en los asuntos de la comunidad. A través de retículas que destacaban los vínculos inextricables entre la autoridad pastoral y el poder terrateniente, la figura del misionero se transformó de la benevolente madre-padre del pasado en el amo opresor del presente. Por úl-

${ }^{42}$ Dube, Stitches on Time, op. cit., capítulo 3.

${ }^{43}$ Brunk, "Balodgahan Village", op. cit. Véase también John Allen Lapp, The Mennonite Church in India, Scottdale, Herald Press, 1972.

${ }^{44}$ Para mayores detalles sobre tal resistencia -y sus múltiples implicaciones-, véase Dube, Stitches on Time, op. cit., sobre todo los capítulos 2 y 3. 
timo, la afirmación de independencia de la congregación de Bisrampur implicó una defensa de los vínculos "paternalistas" que los habían unido con los misioneros por medio de complejos diseños de dependencia y control. Aquí, la deferencia con los misioneros era en parte por autopreservación y en parte por una extracción calculada de tierra, empleo y caridad. Los conversos trabajaron en la retórica misionera y nacionalista en su práctica, e interpretaron su desafío a la autoridad misionera en un lenguaje cristiano mediante expresiones de una gubernamentalidad evangélica. Aunque no de forma tan dramática, los habitantes de Balodgahan también negociaron la autoridad misionera, y sus representaciones cotidianas se inspiraban en el poder pastoral y ritual de diversas maneras. ${ }^{45}$ Junto con los evangelistas euroamericanos, los habitantes de Bisrampur y Balodgahan expresaron los términos y las texturas del imperio, la nación y la modernidad. En cada caso, la aldea - como institución e imaginación, como problema y proceso, como lugar y práctica, y como predicamento y posibilidad-desempeñó un papel clave.

\section{Legalidades e ilegalidades}

Los enredos evangélicos tenían un contexto más amplio, relacionado con el dominio imperial. A mediados del siglo XIX, después de adquirir los territorios del rajá de Nagpur, los británicos crearon las Provincias Centrales y reconstituyeron la administración de esta gran provincia de la India británica. Hacia mediados del siglo xx, la recaudación de rentas agrarias y el mantenimiento del orden público constituyeron, en conjunto, la piedra angular del interés del Estado colonial en la India central. ${ }^{46} \mathrm{El}$ Estado colonial obtuvo acceso a sus súbditos principalmente a través de redes ordenadas de relaciones de propiedad y los mecanismos del orden público. Además, con estas medidas también intentó contener y controlar, definir y disciplinar, y normalizar y naturalizar a los pueblos indios (del

\footnotetext{
${ }^{45}$ Brunk, "Balodgahan Village", op. cit.

${ }^{46}$ Dube, Untouchable Pasts, op. cit., capítulos 3 y 4.
} 
centro del país). Estas matrices enmarcaron y expresaron nociones sobre la persona y el súbdito. ${ }^{47}$

La aldea fue crítica para tales procesos al menos de dos maneras. Por un lado, el sistema de rentas basado en el acuerdo con los malguzares tendía a ver a las personas como parte de los acuerdos de propiedad y tenencia de la tierra en la comunidad aldeana. Aquí se encuentran expresiones interesantes de la posesión, la propiedad y la persona, que convierten a la aldea a la vez en un individuo colectivo y en un colectivo individuado. Incrustadas en matorrales de informes sobre ingresos, documentos relativos a acuerdos, casos civiles y decretos judiciales, tales cuestiones aún aguardan una evaluación sostenida en el estudio académico antropológico e histórico. Por otro lado, las formas y las modalidades que estructuran la noción de la persona en los juzgados penales, ayudados una vez más por procedimientos de vigilancia, estaban más delineadas pero no eran menos complejas. En este escenario, las prácticas y los discursos del derecho colonial expresaban y heredaban una noción de la persona como "individuo". Aquí el individuo se veía como un "todo" integrado, desprendible de la matriz de relaciones sociales y definible en términos de un conjunto discreto de necesidades. Tal noción no es sólo normativa sino también profundamente legislativa. Además de una construcción de regímenes modernos de poder, es una manera de distinguir patologías, extender el control sobre ámbitos difusos e íntimos de la vida social y un método para producir súbditos normalizados. No obstante, al mismo tiempo la aldea no estaba completamente ausente de este terreno, pues el derecho colonial, al definir un delito, podía prestar atención a esquemas de entendimiento y acción “indígenas”, incluso basándose en patrones de normas y prácticas "locales" de rango y honor, y

${ }^{47}$ Las breves consideraciones de esta sección se derivan de un proyecto en curso más amplio sobre la interacción entre el derecho "colonial-moderno" y las legalidades "populares-coetáneas", que aborda nociones definidas sobre el delito y la criminalidad, la autoridad y la moralidad, y la propiedad y la persona, en el desentrañamiento del género y la casta, el parentesco y el vecinazgo, y el Estado-nación y el sujeto-ciudadano en la India central. El estudio se basa principalmente en una extensa investigación en archivos ricos y raros en la India central que contienen registros (en hindi, chhattisgarhi e inglés) de controversias aldeanas que se adjudicaron en cortes coloniales entre 1915 y 1955, así como en otros materiales jurídicos oficiales y extraoficiales. 
de casta y género; de hecho, con frecuencia parecía que la ley aprehendía tales matrices como unidas a los contornos de las comunidades aldeanas. Es decir, al adjudicar un delito, el establecimiento de la culpa y las construcciones de la persona en el derecho colonial no podían escapar a la presencia de la aldea. ${ }^{48}$

Todo ello lleva a otra pregunta: ¿cómo debemos entender el lugar de la ley y de la aldea -y de las configuraciones del Estado y la comunidad- en expresiones de legalidades e ilegalidades populares? Desde hace tiempo, muchas fábulas se han derivado de esa oposición simple pero decisiva entre los procesos de disputa populares-tradicionales-aldeanos (inmutables) y los sistemas jurídicos coloniales-modernos-estatales (dinámicos). Por supuesto, hoy se cuentan nuevas historias sobre diferentes mecanismos y matrices jurídicos. ${ }^{49} \mathrm{Al}$ mismo tiempo, los recuentos que desarrollan las imbricaciones y las implicaciones del derecho moderno y las legalidades populares siguen siendo una rareza en el escenario del sur de Asia. ${ }^{50} \mathrm{El}$ problema sólo se complejiza por la suposición de una división bastante hermética entre el Estado colonial (y las instituciones modernas) y las comunidades indígenas (y las aldeas tradicionales) que apuntala hasta las más imaginativas consideraciones de estos sujetos. ${ }^{51}$

${ }^{48}$ Aquí observamos preguntas que requieren una evaluación cuidadosa y crítica mucho más profunda como parte de mi investigación más amplia sobre las legalidades y las ilegalidades, ya mencionada, pero también temas que he planteado anteriormente en Stitches on Time, op. cit., sobre todo en los capítulos 3 y 4.

${ }^{49}$ Por ejemplo, Erin P. Moore, “Gender, Power, and Legal Pluralism”, American Ethnologist, vol. 20, núm. 3, 1993, pp. 522-542, y Leela Dube, "Conflict and Compromise: Devolution and Disposal of Property in a Matrilineal Muslim Society", Economic and Political Weekly, vol. 29, núm. 21, 1994, pp. 1273-1284.

${ }^{50}$ Considérese Upendra Baxi, “'The State’s Emissary’: The Place of Law in Subaltern Studies”, en Partha Chatterjee y Gyan Pandey (eds.), Subaltern Studies VII. Writings on South Asian History and Society, Delhi, Oxford University Press, 1992, pp. 247-264; Dube, Untouchable Pasts, op. cit., e Ishita Banerjee-Dube, “Taming Traditions: Legalities and Histories in Twentieth century Orissa”, en Gautam Bhadra, Gyan Prakash y Susie Tharu (eds.), Subaltern Studies X: Writings on South Asian History and Society, Delhi, Oxford University Press, 1999, pp. 98-125. Véase también Veena Das, "Subaltern as Perspective", en Ranajit Guha (ed.), Subaltern Studies vi: Writings on South Asian History and Society, Delhi, Oxford University Press, 1989, pp. 310-324.

${ }^{51}$ Discuto el sitio crucial de las metáforas de gobierno colonial y símbolos del Estado en la estructuración del dominio de las castas, la constitución de la comunidad y la creación de legalidades y moralidades alternativas en Untouchable Pasts, op. cit. Veáse también Dube, Stitches on Time, op. cit., y Banerjee-Dube, “Taming Traditions”, op. cit. 
Abordar críticamente y exceder cuidadosamente ordenamientos tan aceptados significa explorar las expresiones sobresalientes pero cambiantes de la ley estatal y la dinámica aldeana, considerando que juntas moldean los términos y las texturas de las ilegalidades y las legalidades populares. Comenzaré con el tema del estatus mismo del archivo legal y cómo debemos leer sus múltiples registros, sobre todo cuando abordamos las controversias que escuchó el Tribunal Supremo y que forman la base de mis argumentos. Resulta de vital importancia reconocer que estas controversias se ubicaron en el ámbito de lo familiar y lo cotidiano en la vida aldeana, mientras que en el ámbito judicial del distrito colonial se convirtieron en "casos penales". Los asuntos en cuestión transitaron y unieron ambos entornos. ${ }^{52}$ Ahora, la riqueza exacta de los materiales de archivo implica que las controversias pueden abordarse y comprenderse de diferentes maneras. Al mismo tiempo, mi apuesta interpretativa concierne a la resignación de que, sean cuales sean los procedimientos de lectura que adoptemos, sería analíticamente fatal dejar de lado la interacción formativa entre los protocolos del orden normativo del derecho y los procesos de significación en las relaciones aldeanas que están en el corazón de las controversias.

Este intercambio constitutivo pone en primer plano que las hechuras de las legalidades y las ilegalidades entretejen inextricablemente lo colonial y lo vernáculo, la ley de la comunidad y el Estado y la aldea. Aquí se encuentra el desentrañamiento de las relaciones de poder estructuradas por la casta, el parentesco y el género, pero siempre afectadas por el derecho colonial y las jerarquías aldeanas. ${ }^{53}$ En este escenario, los signos y los símbolos, y los términos y las prácticas del derecho colonial moderno aparecen a la vez como legalidades ajenas, protocolos de establecimiento y venganza, y recursos que despliegan selectivamente los pueblos aldeanos para definir nuevas patologías y crear legalidades inéditas en el ámbito de la vida

${ }^{52}$ Para consultar las maneras complejas en que las controversias fueron a horcajadas entre los ámbitos cotidianos y los teatros coloniales, y los diversos problemas que se enfrentaron en las lecturas de este archivo, véase Dube, Stitches on Time, op. cit., capítulos 3 y 4.

${ }^{53}$ Idem. 
cotidiana. ${ }^{54}$ Tal interpretación de las ilegalidades cotidianas y las legalidades populares, a través del trabajo con el derecho estatal, subyacen tras articulaciones nuevas de género, transformaciones críticas de parentesco, formulaciones frescas de orden $y$, de hecho, novedosas interpretaciones de la aldea.

\section{Coda}

Consideradas como un concepto y una entidad, hay variedades de aldeas que han pasado a través de estas páginas. Los múltiples mapeos y las agudas ambivalencias que rodean a la aldea han ayudado a reflejar el pasado y el presente de la antropologíasociología de India, incluidos los vínculos disciplinarios con el Estado y la nación, que se observan de diferentes formas. Las articulaciones definidas y las expresiones discretas de las aldeas en Chhattisgarh han servido como una suerte de prisma que refleja y refracta los diseños y los detalles del imperio y a modernidad, del proyecto misionero y un cristianismo vernáculo, del derecho (y el orden) colonial y las legalidades (e ilegalidades) cotidianas.

¿Resulta sorprendente algo de esto? No si vemos las aldeas como interpretadas y habitadas a través de las convenciones de significado y la práctica de mundos académicos y sociales, mundos que se unen y se separan. No si consideramos que la aldea insinúa más que un objeto de entendimiento, una condición del saber, el primero, deseo transformado por el último, imperativo. ¿Acaso éstas puedan ser lecciones que recordar conforme las aldeas —en muchos, muchos ámbitos-se siguen encontrando, perdiendo y recuperando?

\section{Traducción del inglés: María Capetillo}

Dirección institucional del autor:

Centro de Estudios de Asia y África

El Colegio de México

Camino al Ajusco 20

${ }^{54}$ Idem. 
Pedregal de Sta. Teresa

10740, México, D.F.

sdube@colmex.mx

\section{Bibliografía}

BAJPAI, David, "My Father - Rev. Simon Ramnath Bajpai”, texto mecanografiado, 84-9b Bio 52, Webster Groves, Eden Archives and Library, 1945.

BanerJee-Dube, Ishita, "Taming Traditions: Legalities and Histories in Twentieth century Orissa”, en Gautam Bhadra, Gyan Prakash y Susie Tharu (eds.), Subaltern Studies X: Writings on South Asian History and Society, Delhi, Oxford University Press, 1999, pp. 98-125.

BAXI, Upendra, “'The State's Emissary': The Place of Law in Subaltern Studies", en Partha Chatterjee y Gyan Pandey (eds.), Subaltern Studies VII. Writings on South Asian History and Society, Delhi, Oxford University Press, 1992, pp. 247-264.

BRUnK, A. C., "Balodgahan Village", manuscrito IV-17-21, caja 28, Charitable Institutions, f. 1, Goshen, Archives of the American Mennonite Church, sin fecha.

CoHn, Bernard, "History and Anthropology: The State of Play", Comparative Studies in Society and History, vol. 22, núm. 2, 1980, pp. 198-221.

ComarofF, John y Jean Comaroff, Ethnography and the Historical Imagination, Boulder, Westview, 1992.

Contributions to Indian Sociology, edición especial, vol. 12, núm. 1, 1978.

DAs, Veena, "Subaltern as Perspective", en Ranajit Guha (ed.), Subaltern Studies VI: Writings on South Asian History and Society, Delhi, Oxford University Press, 1989, pp. 310-324.

DiRKs, Nicholas, Castes of Mind: Colonialism and the Making of Modern India, Princeton, Princeton University Press, 2001.

Dube, Leela, "Conflict and Compromise: Devolution and Disposal of Property in a Matrilineal Muslim Society", Economic and Political Weekly, vol. 29, núm. 21, 1994, pp. 1273-1284.

Dube, S. C., India's Changing Villages: Human Factors in Community Development, Londres, Routledge and Kegan Paul, 1958.

Dube, S. C., Indian Village, Londres, Routledge and Kegan Paul, 1955.

DubE, S. C., The Kamar, Lucknow, Universal, 1951. 
Dube, Saurabh, After Conversion: Cultural Histories of Modern India, Nueva Delhi, Oxford University Press, 2010.

Dube, Saurabh, “Anthropology, History, Historical Anthropology”, en Saurabh Dube (ed.), Historical Anthropology, Nueva Delhi, Oxford University Press, 2007, pp. 1-73.

Dube, Saurabh, "Conversion to Translation: Colonial Registers of a Vernacular Christianity", en Saurabh Dube (ed.), Enduring Enchantments, edición especial de South Atlantic Quarterly, vol. 101, núm. 4, 2002, pp. 807-837.

Dube, Saurabh, Stitches on Time: Colonial Textures and Postcolonial Tangles, Durham, Duke University Press, 2004.

Dube, Saurabh, "Ties that Bind: Tribe, Village, Nation, and S. C. Dube", en Patricia Uberoi, Satish Deshpande y Nandini Sundar (eds.), Disciplinary Biographies: Essays in the History of Indian Sociology and Anthropology, Delhi, Permanent Black, 2007, pp. 444-495.

Dube, Saurabh, Untouchable Pasts: Religion, Identity, and Power among a Central Indian Community, 1780-1950, Albany, State University of New York Press, 1998.

Dube, Saurabh, "Witnessing Lives: Conversion and Life-history in Colonial Central India", en Ishita Banerjee-Dube y Saurabh Dube (eds.), Ancient to Modern: Religion, Power, Community, Nueva Delhi, Oxford University Press, 2009, pp. 259-290.

Dube, Saurabh e Ishita Banerjee-Dube, "Spectres of Conversion: Transformations of Caste and Sect in India”, en Rowena Robinson y Sathianathan Clarke (eds.), Religious Conversion in India: Modes, Motivations, and Meanings, Nueva Delhi, Oxford University Press, 2003, pp. 222-254.

Dumont, Louis y David F. Pocock, "For a Sociology of India", Contributions to Indian Sociology, vol. 1, 1957, pp. 7-22.

Fabian, Johannes, Time and the Other: How Anthropology makes its Object, Nueva York, Columbia University Press, 1983.

InDEN, Ronald, Imagining India, Oxford, Basil Blackwell, 1990.

JAIN, Ravindra K., "Social Anthropology of India: Theory and Method", en Indian Council of Social Science Research, Survey of Research in Sociology and Social Anthropology, 1969-1979, Nueva Delhi, Satvahan, 1985, pp. 1-50.

LAPP, John Allen, The Mennonite Church in India, Scottdale, Herald Press, 1972.

Leonardo, Micaela di, Exotics at Home: Anthropologies, Others, and American Modernity, Chicago, University of Chicago Press, 2000. 
LuTz, Catherine y Jane Collins, Reading National Geographic, Chicago, University of Chicago Press, 1993.

Madan, T. N., Pathways: Approaches to the Study of Society in India, Delhi, Oxford University Press, 1994.

MarriotT, McKim (ed.), Village India: Studies in the Little Community, Chicago, University of Chicago Press, 1955.

MilleR, "Survey of Bisrampur", Bisrampur Malguzari File, Webster Groves, Eden Archives and Library, 1925.

Mines, Diane y Nicolas Yazgi (eds.), Village Matters: Relocating Villages in the Contemporary Anthropology of India, Nueva Delhi, Oxford University Press, 2010.

Moore, Erin P., "Gender, Power, and Legal Pluralism”, American Ethnologist, vol. 20, núm. 3, 1993, pp. 522-542.

Paul, M. M., "Autobiography of M. M. Paul, Head Catechist at Mahasamund", texto mecanografiado, 84-9b Bio 52, Webster Groves, Eden Archives and Library, sin fecha, s.a.

Redfield, Robert, Peasant, Society, and Culture, Chicago, University of Chicago Press, 1956.

SABERWAL, Satish, "Sociologists and Inequality in India: The Historical Context”, Economic and Political Weekly, vol. 14, núm. 7-8, 1979, pp. 243-254.

SEYbOLD, Theodore, “The Reverend Ramnath Simon Bajpai”, texto mecanografiado, 84-9b Bio 52, Webster Groves, Eden Archives and Library, sin fecha.

Srinivas, M. N. (ed.), India's Villages, Bombay, Media Promoters and Publishers, 1955.

SRINIVAS, M. N., Religion and Society among the Coorgs of South India, Oxford, Clarendon Press, 1952.

SRINIVAS, M. N., Social Change in Modern India, Berkeley, University of California Press, 1966.

SRInIVAs, M. N., The Remembered Village, Delhi, Oxford University Press, 1976.

Thomas, Nicholas, Out of Time: History and Evolution in Anthropological Discourse, Ann Arbor, University of Michigan Press, 1996.

Trouillot, Michel-Rolph, "Anthropology and the Savage Slot: The Poetics and Politics of the Otherness", en Richard Fox (ed.), Recapturing Anthropology: Working in the Present, Santa Fe, School of American Research Press, pp. 17-44.

Trouillot, Michel-Rolph, "North Atlantic Universals: Analytical Fictions, 1492-1945”, en Saurabh Dube (ed.), Enduring Enchantments, edición especial de South Atlantic Quarterly, vol. 101, núm. 4, 2002, pp. 839-858. 